\title{
Rice UBC13, a candidate housekeeping gene, is required for K63-linked polyubiquitination and tolerance to DNA damage
}

\author{
Yuepeng Zang ${ }^{1}$, Qian Wang ${ }^{1}$, Chenyu Xue ${ }^{1}$, Mengnan Li ${ }^{1}$, Rui Wen ${ }^{1,2}$ and Wei Xiao ${ }^{1,2^{*}}$
}

\begin{abstract}
While plant growth and reproduction is dependent on sunlight, UV irradiation from sunlight is one of the major genotoxic stresses that threaten plant survival and genome stability. In addition, many environmental chemicals can also damage the plant genome. In yeast and mammalian cells protection against the above genome instability is provided by an error-free DNA-damage tolerance (DDT) pathway, which is dependent on Ubc13-mediated K63-linked polyubiquitination of the proliferating cell nuclear antigen (PCNA). In this study, we isolated the UBC13 gene from rice and characterized its functions. Expression of OsUBC13 can protect a yeast $u b c 13$ null mutant against spontaneous and environmental DNA damage. Furthermore, OsUbc13 physically interacts with human Ubc13 partners Mms2 and Uev1A, and catalyzes K63 polyubiquitination in vitro. These observations collectively suggest that the K63 polyubiquitination is conserved in rice, and that OsUBC13 may be involved in DDT and other cellular processes. In addition, OsUBC13 is constitutively expressed at a high level even under various stress conditions, suggesting that it is a housekeeping gene.
\end{abstract}

Keywords: Rice, Ubc13, K63-linked polyubiquitination, DNA-damage tolerance, Signal transduction

\section{Background}

Ubiquitination is an essential protein posttranslational modification (PTM) process found in all eukaryotic organisms. This modification by a 76 amino acid ubiquitin $(\mathrm{Ub})$ determines protein turnover, regulation and molecular function, and provides a complex regulation of various biological processes (Hershko and Ciechanover 1998). The most well-understood ubiquitination pathways are the ubiquitin-proteasome system (UPS) that mediates protein degradation (Hochstrasser 1996). UPS has been reported to be involved in almost all aspects of plant biology, including phytohormone signalling (auxin, gibberellins, jasmonic acid etc.), plant morphogenesis, immune response, self-incompatibility, chromatin modification and epigenetic regulation (Vierstra 2009). Ubiquitination, as an enzymatic cascade, is catalyzed by members of three enzyme families: ubiquitin-activating enzyme (Uba or E1), ubiquitin-

\footnotetext{
* Correspondence: wei.xiao@usask.ca

${ }^{1}$ College of Life Sciences, Capital Normal University, Beijing, China 100048

${ }^{2}$ Department of Microbiology and Immunology, University of Saskatchewan, Saskatoon, SK, Canada S7N 5E5
}

conjugating enzyme (Ubc or E2) and ubiquitin ligase (Ubl or E3) enzymes. Before conjugation takes place, the initial activation of ubiquitin is performed by E1 in an ATP-dependent manner. The activated ubiquitin is then transferred from E1 to an active-site cysteine of E2 via a trans-esterification reaction. Finally, a Ubloaded E2 selectively interacts with a cognate E3 that recruits a specific substrate. The $\mathrm{C}$-terminal glycine of $\mathrm{Ub}$ is linked to the $\epsilon$-amino group lysine residue of either the target protein or a previously attached $\mathrm{Ub}$ (Hershko and Ciechanover 1998; van Wijk and Timmers 2009). Unlike HECT (homologous to E6-AP C terminus) E3s that first accept the activated Ub prior to substrate modification (Rotin and Kumar 2009), RING (really interesting new gene) and U-box E3s act as a platform and provide an optimal conformation for the $\mathrm{Ub}$ transfer while binding both the substrate and Ub-loaded E2 (Ardley and Robinson 2005; Deshaies and Joazeiro 2009).

In this enzymatic cascade, the highly-conserved E2 family that couples the activation of Ub to the conjugation event appears to play a central role. E2s directly influence which surface lysine residue in $\mathrm{Ub}$ will be 
attached to the incoming $\mathrm{Ub}$, which affects the destiny of the substrate (Burroughs et al. 2008; Pickart 2000; Pickart and Fushman 2004). In the UPS pathway, the target protein is modified by a Lys48 (K48)-linked poly-Ub chain that is recognized and degraded by $26 \mathrm{~S}$ proteasome (Hershko and Ciechanover 1998). In Arabidopsis this process is catalyzed by at least $37 \mathrm{E} 2 \mathrm{~s}$ and accompanied by potentially over 1,400 different E3s to recognize different substrates in various biological pathways (Kraft et al. 2005; Vierstra 2009). In contrast, only one E2, Ubc13, has been reported to be capable of catalyzing K63-linked poly-Ub chain assembly in eukaryotes, from unicellular yeasts to human, and this non-canonical poly-Ub chain assembly requires a heterodimeric E2 complex consisting of Ubc13 and a Ubc-E2 variant (Uev) (Hofmann and Pickart 1999; McKenna et al. 2001). In Saccharomyces cerevisiae, Ubc13-Uev (Mms2) complex coupled with the E3 Rad5 modifies proliferating cell nuclear antigen (PCNA) at its K164 residue with K63-linked poly-Ub chain, which promotes error-free DNA-damage tolerance (DDT, also known as post-replication repair, PRR) as one of two means of bypassing replication-blocking lesions (Broomfield et al. 1998; Hoege et al. 2002; Hofmann and Pickart 1999; Ulrich and Jentsch 2000). Another lesion bypass method is called translesion DNA synthesis (TLS), which utilizes non-essential DNA polymerases including Poln (Rev3+Rev7), Pol $\zeta$, and Rev1 (Ulrich and Jentsch 2000; Xiao et al. 2000). It is now clear that the K164 residue of PCNA is monoubiquitinated by the E2-E3 complex Rad6-Rad18, which promotes TLS, whereas its sequential K63-linked polyubiquitination by Mms2-Ubc13-Rad5 promotes error-free DDT (Hoege et al. 2002; Pastushok and Xiao 2004). Interestingly, the PCNA-K164 residue can also be SUMOylated by the E2E3 complex Siz1-Ubc9 (Hoege et al. 2002; Stelter and Ulrich 2003). Consequently, the SUMOylated PCNA can recruit the DNA helicase Srs2 to stalled replication forks to prevent unwanted homologous recombination (Papouli et al. 2005; Pfander et al. 2005).

Plants, as sessile lifestyle organisms, have to face numerous environmental stresses including genotoxic stress, such as UV irradiation and chemical pollutants. This wide range of stresses threatens plants survival, reduces crop yields and food quality, and compromises world food security. Although there are some reports on genes involved in plant DDT, most describe TLS polymerases (Anderson et al. 2008; Curtis and Hays 2007; Garcia-Ortiz et al. 2004; Sakamoto et al. 2003; Takahashi et al. 2005) and a few on error-free DDT genes from Arabidopsis (Chen et al. 2008; Wen et al. 2006; Wen et al. 2008), with little elucidation on the mechanism. Surprisingly, to the best of our knowledge, there is no report on the isolation and characterization of genes involved in DDT in crop plants. In this study, the rice
$U B C 13$ gene was isolated and functionally characterized. It was found that $O s U B C 13$ is able to functionally complement the yeast $u b c 13$ null mutant and that OsUbc13 protein can interact with Uevs from other species and mediate K63-linked polyubiquitination in vitro, suggesting that it may play similar roles in rice. It was also found that both the transcript and protein levels of OsUbc13 remain stable during plant growth and development, as well as facing various biotic and abiotic stresses. We argue that OsUBC13 is a candidate housekeeping gene and its expression may be used as a useful internal control for the study of other rice genes or gene products.

\section{Results}

Identification and sequence analysis of the OsUBC13 gene To identify the rice $U b c 13$ gene, a pair of highly conserved Arabidopsis $U B C 13$ genes was used to BLAST the rice genomic database on NCBI. Only one hypothetical gene (LOC_Os01g48280) with a high degree of sequence identity was found in the rice genome; it was named OsUBC13. OsUBC13 encodes a protein of 153 amino acids with an estimated molecular weight of approximately $17 \mathrm{kDa}$. The amino acid sequence comparison of OsUbc13 with its counterparts in some other species reveals that it differs from two Arabidopsis Ubc13s, AtUbc13A and AtUbc13B, by 5 and 3 amino acids, respectively (Figure 1a). OsUbc13 also shows a high degree of conservation with Ubc13s from other eukaryotic organisms. Moreover, critical amino acid residues defined in hUbc13, including Cys87 in the active site for Ub thioester formation (McKenna et al. 2001), Met64 for the physical interaction with RING finger E3 ligases (Wooff et al. 2004), Glu55, Phe57 and Arg70, which form a "pocket" that determine the specific interaction with Mms2 (Pastushok et al. 2005), as well as a Q100 residue as the target of bacterial OspI deamidation (Sanada et al. 2012), are all conserved in OsUbc13 (Figure 1a). Genomic DNA sequence analysis as shown in Figure $1 \mathrm{~b}$ indicate that yeast, plant and mammalian $U B C 13 \mathrm{~s}$ are derived from the same origin in evolution, since they also share some intron-exon junctions. To further strengthen this argument, we performed phylogenetic analysis of Ubc13 and its closely related Ubcs from several model organisms ranging from yeast to plants to human. As shown in Additional file 1: Figure S1, all Ubc13s stay in the same branch away from other most closely related Ubcs, confirming that UBC13 orthologs from entire eukaryotes are derived from the same origin. Although AtUBC13A and AtUBC13B share the same intron-exon junctions, several of their intron lengths are different. Interestingly, all except two OsUBC13 introns are different from that of $A t U B C 13 B$, consistent with the amino acid alignment indicating that 

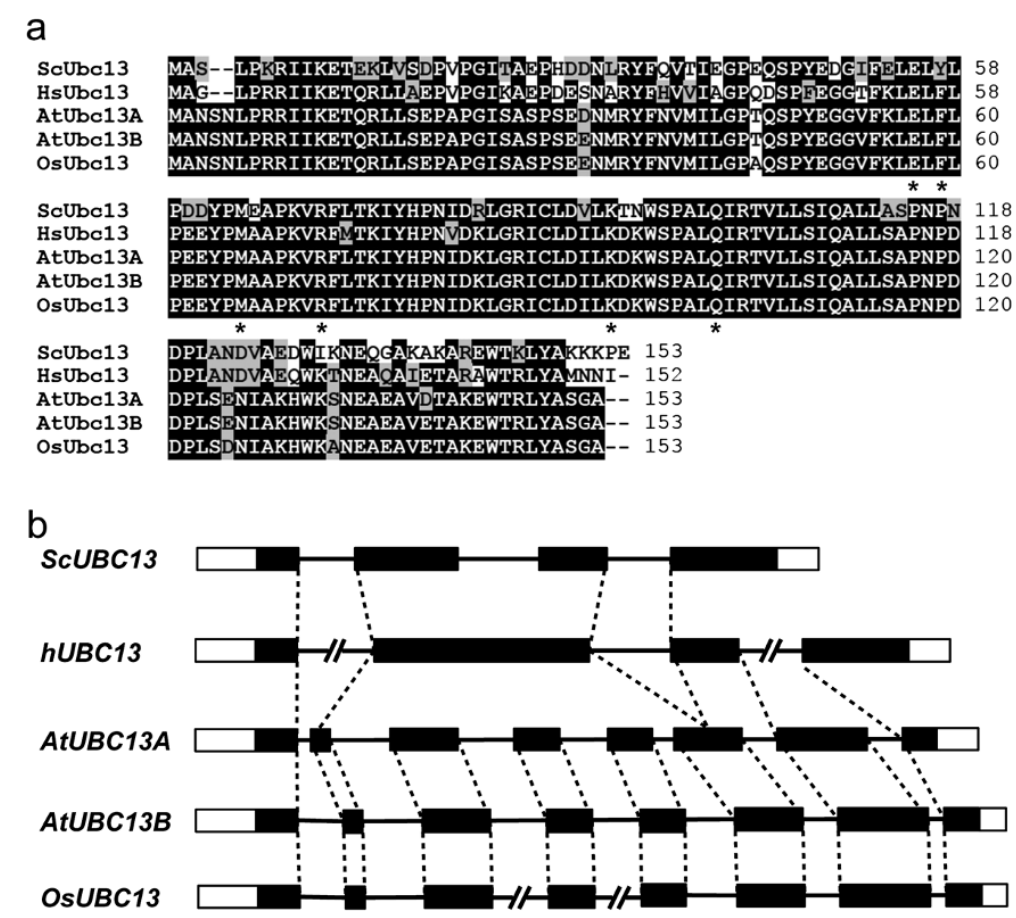

Figure 1 Sequence analysis of OsUbc13 and its gene structure. a Protein sequence alignment of OsUbc13 and Ubc13s from three other organisms. The sequences were aligned and edited using the BioEdit program version 7.0.9. Identical residues are highlighted with black while conserved residues are grey. Critical residues for Ubc13 functions are indicated with asterisks underneath the residue. $\mathbf{b}$ Genomic organization of UBC13 genes from different organisms. Open boxes, untranslated region; filled boxes, coding regions; lines, introns; broken lines, introns longer than their proportional representation; dotted lines, identical intron-exon alignment between different UBC13 genes. All intron-exon junctions are identical among AtUBC13A, AtUBC13B and OsUBC13.

OsUBC13 is more closely related to $A t U B C 13 B$ than to AtUBC13A.

\section{OsUBC13 Functionally complements yeast ubc13 null mutant}

Yeast $U B C 13$ plays a critical role in protecting cells from mutagenesis and cell death caused by DNA-damaging agents (Brusky et al. 2000). In order to determine whether OsUBC13 could functionally complement the error-free PRR defect in the yeast $u b c 13$ null mutant, we performed yeast killing and spontaneous mutagenesis assays. As shown in Figure 2a, expression of OsUBC13 from a yeast two-hybrid vector rescued the $u b c 13$ null mutant from methyl methanesulfonate (MMS)-induced killing by up to 100 -fold. It was noticed that while OsUBC13 protects yeast $u b c 13$ mutant cells to a full wild-type level after 20- and 40-minute treatment, only partial complementation was achieved after 60-minute treatment. We suspect that it was due to either the nature of heterologous expression, or plasmid loss since cells were grown in the absence of selection shortly before and during MMS treatment. To further assess the OsUBC13 function in yeast cells, a semi-quantitative serial dilution assay was performed in the presence of a few representative DNA-damaging agents. As shown in Figure $2 \mathrm{~b}$, OsUBC13 rescues the yeast $u b c 13$ mutant from killing by MMS (alkylation damage), 4-nitroquinoline 1oxide (4NQO, bulky lesions) and UV irradiation to a level comparable to that in wild-type cells, whereas the vector alone has no rescuing effect.

Another complementary assay was based on astonishing phenotypes of an mms 2 (Broomfield et al. 2001) or $u b c 13$ (Brusky et al. 2000) mutant with massive increase in the spontaneous mutagenesis rate, providing strong evidence that $U B C 13$ plays a critical role in maintaining genomic stability of host cells. Indeed, deletion of $U B C 13$ causes a 25 -fold increase in the spontaneous mutation rate; when these $u b c 13$ mutant cells were transformed with a plasmid containing OsUbc13, the spontaneous mutation rate was reduced to about fivefold (Table 1). The residual increase in spontaneous mutagenesis above the wild type level was largely due to plasmid loss in a certain fraction of transformed cells (established to be $10-20 \%$, data not shown) under non-selective conditions. Taken together, the above two sets of experiments allow us to conclude that OsUbc13 functionally complements the defects of the yeast $u b c 13$ null mutant in the error-free PPR pathway. 
a

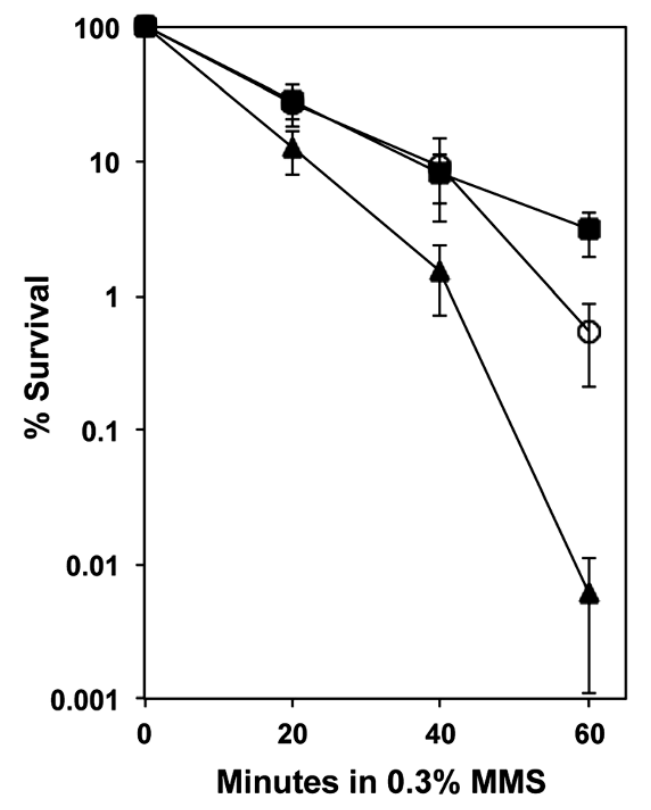

b

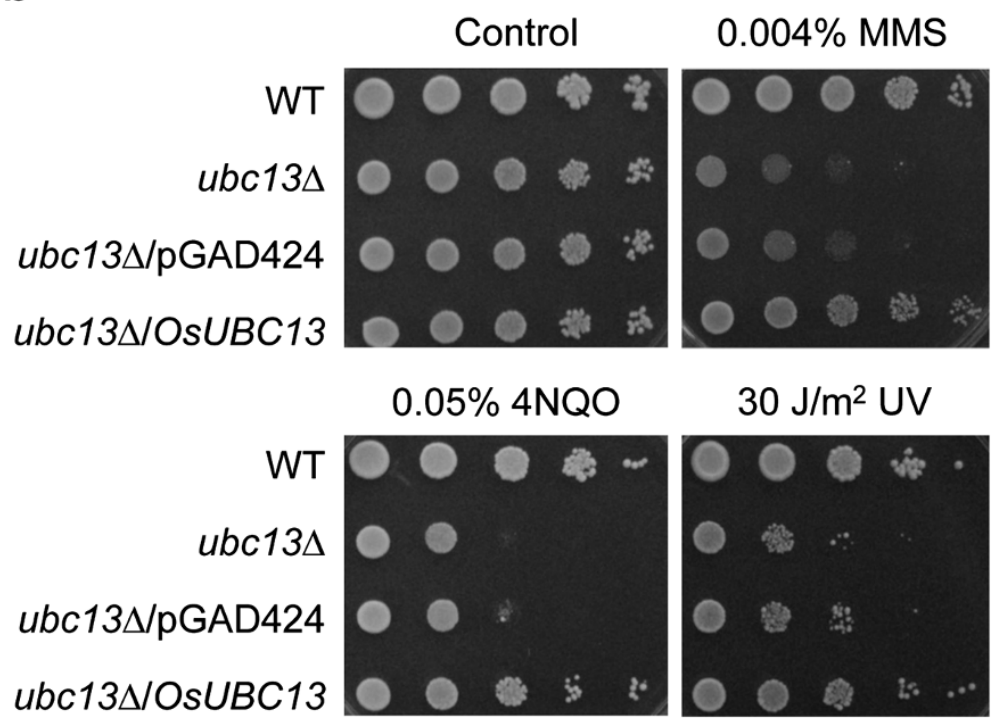

Figure 2 Functional complementation of yeast ubc13 null mutant by OsUBC13. a Protection of the $u b c 13$ mutant by OsUBC13 from MMS-induced DNA damage by a liquid killing experiment. Cells were grown in 'rich medium 30 min prior to the treatment. Results are from the average of at least three independent experiments with standard deviations. (-) HK578-10D (wild type); ( $\mathbf{\Delta}$ ) WXY904 (ucb13) and (O) WX904 transformed with pGAD-OsUbc13. b Protection of the ubc13 mutant by OsUBC13 from representative DNA-damaging agents by a serial dilution assay. Cells were grown overnight in SD selective media, diluted and treated as previously described (Wang et al. 2011). Photographs were taken after 2-day incubation at $30^{\circ} \mathrm{C}$.

OsUbc13 Physically interacts with yeast and human E2 variants

It has been known that, both in yeast and human, the assembly of K63-linked poly-Ub chains requires Ubc13 and a Uev to form a stable heterodimer (Hofmann and Pickart 1999; McKenna et al. 2001; VanDemark et al. 2001). In order to detect this interaction, a yeast two- hybrid assay (Fields and Song 1989) was performed between the human Uev (hUev1A and hMms2), yeast Uev (yMms2) and OsUbc13. Co-expression of $\mathrm{Gal}_{\mathrm{BD}^{-}}$ OsUbc13 with either human Uevs (Figure 3a) or yMms2 (Figure $3 \mathrm{~b}$ ) $\mathrm{Gal}_{\mathrm{AD}}$ fusion enabled cell growth in the minimal medium containing up to $5 \mathrm{mM}$ 1,2,4aminotriazole (3-AT). This is believed to be due to a 
Table 1 Spontaneous mutation rates

\begin{tabular}{|c|c|c|c|}
\hline Strain $^{a}$ & Key alleles & Rate $^{b}\left(\times 10^{-8}\right)$ & Fold increase $^{c}$ \\
\hline DBY747 & Wild type & $1.28 \pm 0.30$ & 1 \\
\hline WXY849 & $u b c 13 \Delta$ & $31.43 \pm 9.48$ & 24.55 \\
\hline WXY849/OsUbc13 & $u b c 13 \triangle \operatorname{Os} U B C 13^{\mathrm{d}}$ & $6.76 \pm 0.33$ & 5.28 \\
\hline
\end{tabular}

${ }^{a}$ All strains are isogenic derivatives of DBY747.

${ }^{\mathrm{b}}$ The spontaneous mutation rates are the average of at least three.

independent experiments with standard deviations.

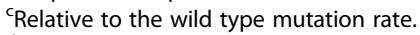

${ }^{\mathrm{d}}$ OsUBC13 cloned in pGAD424, as used in the yeast two-hybrid assay. complex formation that brought $\mathrm{Gal}_{\mathrm{AD}}$ and $\mathrm{Gal} 4_{\mathrm{BD}}$ in proximity at the $P_{G A L 1}$ promoter that resulted in the activation of a cellular $P_{G A L 1}-H I S 3$ reporter gene. All of the above interactions are robust and deemed strong, as none of the negative controls reveal positive interactions under low stringency and many bona fide positive interactions may not survive as low as $1 \mathrm{mM} 3$-AT concentration under the same experimental conditions (data not shown).

To further confirm the yeast two-hybrid results, a glutathione S-transferase (GST)-affinity pull-down assay a

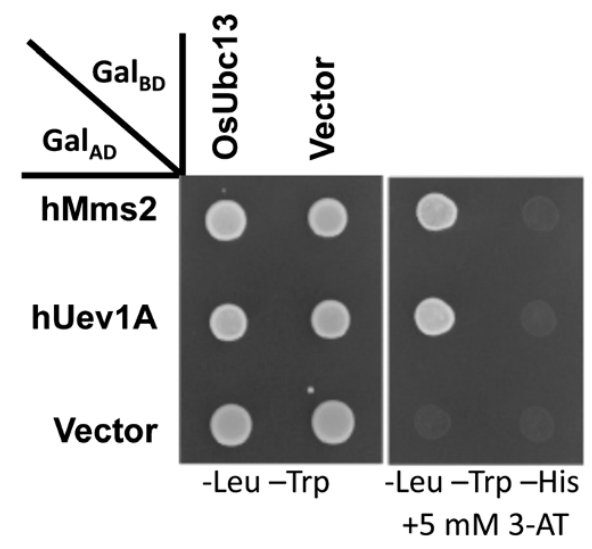

b

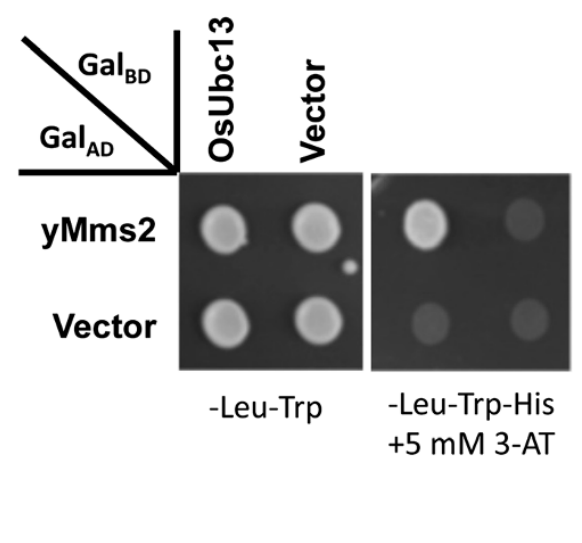

C

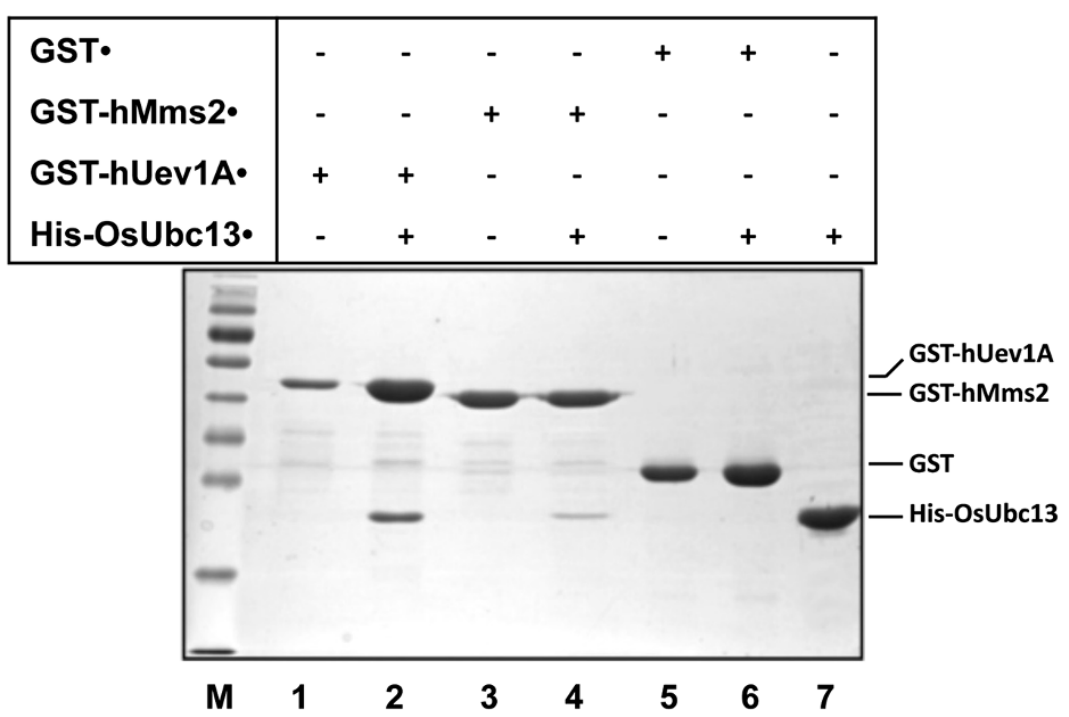

Figure 3 Physical interaction of OsUbc13 with Mms2/Uev1A from other species. a Physical interaction between OsUbc13 and human Mms2/Uev1A in a yeast two-hybrid assay. b Physical interaction between OsUbc13 and yeast Mms2 in a yeast two-hybrid assay. a,b The PJ69-4A transformants carrying one Gal4 $4_{A D}$ (from pGAD424 derivative) and one Gal4 $4_{B D}$ (from pGBT9 derivative) were replicated onto various plates as indicated and incubated for 3 days before being photographed. The result is representative of at least five independent transformants from each treatment. c Protein interactions between OsUbc13 and human Mms2/Uev1A by an affinity pull-down assay. A crude whole-cell extract from co-transformed E. coli cells was incubated with $100 \mu \mathrm{l} \mathrm{GST}$ beads for $2 \mathrm{hr}$ at $4^{\circ} \mathrm{C}$. The beads were then boiled with SDS-PAGE loading buffer for 5 min before SDS-PAGE analysis. GST-hUev1A (Lane 1), GST-hMms2 (Lane 3), GST (Lane 5) and His-OsUbc13 (Lane 7) alone served as controls. pGEX6 (GST) co-transformed with His-OsUbc13 (Lane 6) also served as a negative control. 
was performed (Figure 3c). In E. coli cells co-transformed with plasmids expressing $\mathrm{His}_{6}-\mathrm{OsUbc13}$ and GST-Uev, $\mathrm{His}_{6}$-OsUbc13 could be co-purified by GST-hUev1A (Lane 2) and GST-hMms2 (Lane 4) from total cell lysates. As a negative control, GST alone (Lane 6) was unable to pull-down $\mathrm{His}_{6}$-OsUbc13 under the same experimental conditions. Furthermore, previous yeast complementation experiments indicate that heterologous Ubc13 must physically interact with the endogenous yMms2, which is the prerequisite for the functional complementation (Pastushok et al. 2005). Taken together, these data collectively suggest that all three Uevs from human and yeast can form stable heterodimers with OsUbc13 in vitro and in vivo.

\section{OsUbc13 Mediates K63-linked polyubiquitination with human Uevs in vitro}

Ubc13, like other Ubcs, is able to form a thioester bond with the C-terminal Ub-Gly76 at its active-site Cys residue; however, Ubc13 is the only identified E2 capable of catalyzing K63-linked polyubiquitination (Hofmann and Pickart 1999), and a Uev is absolutely required for this ubiquitin chain assembly. Moreover, the Ubc13-Uev complex is proved to catalyze poly-Ub chain formation through Ub-K63 instead of K48 linkage (Hofmann and Pickart 1999; Hofmann and Pickart 2001; McKenna et al. 2001). In Arabidopsis, both AtUbc13A and AtUbc13B can promote K63-linked poly-Ub chain formation with any of the four AtUev1s (Wen et al. 2008). In order to detect the catalytic ability of the E2 enzyme, OsUbc13, hUev1A and hMms2 were purified for an in vitro ubiquitination assay. As shown in Figure 4,

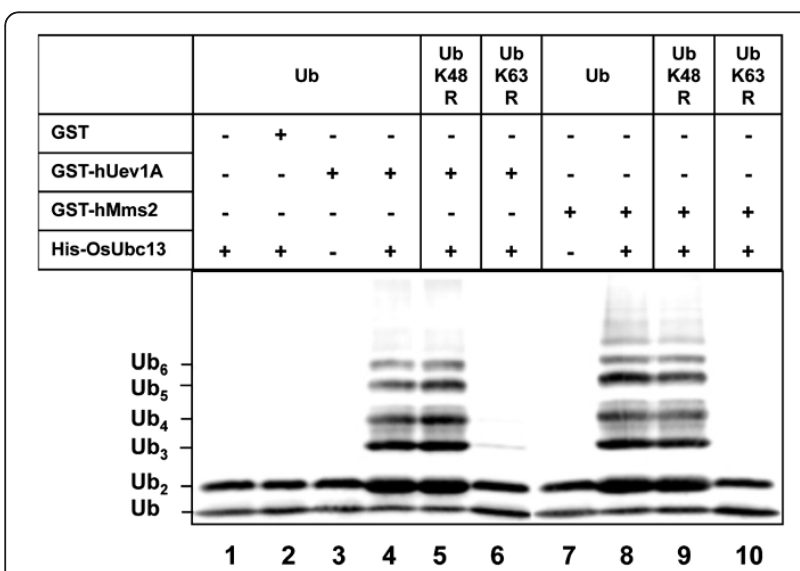

Figure 4 In vitro ubiquitin conjugation assays using purified proteins. After ubiquitination reactions as described, samples were subjected to SDS-PAGE and a western blot using an anti-Ub antibody was preformed to monitor poly-Ub chain formation. The background of spontaneously formed di-Ub in the absence of E2 or Uev (Lanes 1, 3, and 7) is commonly observed in these reactions. Line 2 containing GST and OsUbc13 serves as a negative control to rule out the effect of GST tag.
OsUbc13 (Lane 1), hUev1A (Lane 3) and hMms2 (Lane 7) alone did not promote free poly-ubiquitin chain formation. When both OsUbc13 and a Uev (hUev1A or $\mathrm{hMms} 2$ ) were present in the same reaction, di-Ub, tri-Ub and even longer Ub chains were readily formed (Lanes 4 and 8 ). Moreover, the assembly of free poly-Ub chains was not affected by the Ub-K48R mutation (Lanes 5 and 9); however, the Ub-K63R mutation completely abolished the $\mathrm{Ub}$ chain formation (Lanes 6 and 10). The above results indicate that with the assistance of hUev1A or hMms2, OsUbc13, can catalyze free poly-ubiquitin chain formation through K63 linkage.

\section{OsUbc13 is ubiquitously expressed and does not fluctuate under biotic or abiotic stresses}

Since Ubc13 is mainly involved in stress responses such as DNA damage and innate immunity, it is reasonable to assume that $U B C 13$ expression may be regulated in response to these stresses. Indeed, S. cerevisiae UBC13 is induced when cells are treated with different DNAdamaging agents (Brusky et al. 2000). In contrast, the expression of neither Arabidopsis $U B C 13$ gene appears to fluctuate in different tissues or be altered by biotic or abiotic stresses (Wen et al. 2006). To further investigate the expression profiles of OsUBC13, we performed a cross-database search on the Genevestigator (Hruz et al. 2008). The retrieved data show that the expression of OsUBC13 (LOC_Os01g48280) remains high and remarkably stable during development (Figure $5 \mathrm{a}$ ) and in different tissues (Additional file 2: Figure S2). The differences, if any, fall in experimental variation and are statistically insignificant. Moreover, none of the biotic or abiotic stress treatment induces or suppresses OsUBC13 expression beyond twofold, and the vast majority are even less than 1.2-fold or perhaps within experimental variation (Additional file 3: Figure S3). Hence, the expression pattern of OsUBC13 appears to be comparable to that of AtUBC13 genes.

Although the transcript level of OsUBC13 is relatively stable, it does not rule out the possibility that OsUBC13 may be regulated at the protein level. To further determine the cellular Ubc13 protein level under various stresses, we used the monoclonal antibody 4E11 produced in-house against hUbc13 (Andersen et al. 2005), which shares a very high degree of amino acid sequence identity with OsUbc13 (Figure 1a). A single band of approximately $20 \mathrm{kDa}$ was detected from total rice seedling extract in a western blot analysis, consistent with the predicted OsUbc13 protein size. In this study, abscisic acid (ABA), salicylic acid (SA) and jasmonic acid (JA) were selected to represent hormone treatments, while methyl methanesulfonate (MMS), cisplatin and hydrogen peroxide $\left(\mathrm{H}_{2} \mathrm{O}_{2}\right)$ were chosen to represent DNA damage treatments. As shown in Figure $5 \mathrm{~b}$ and quantitated by 


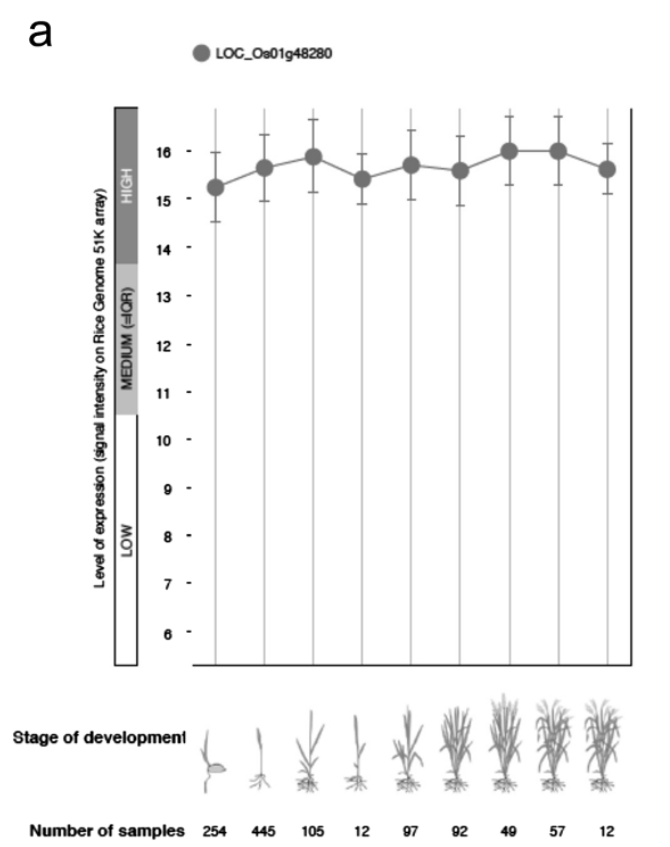

b
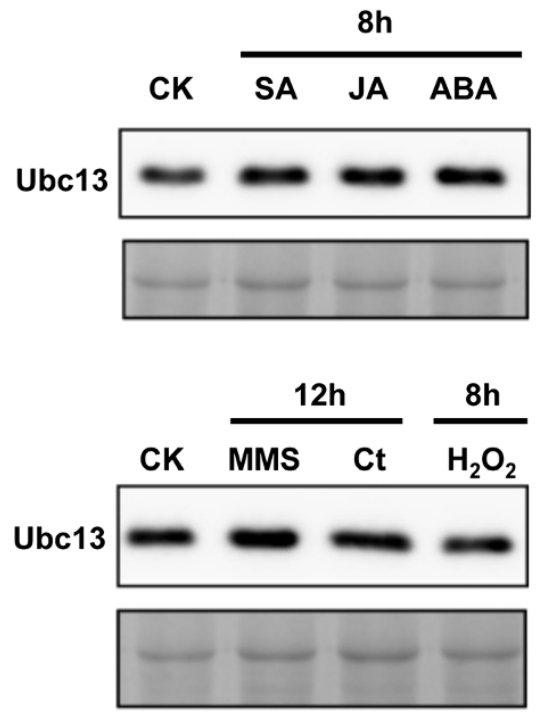

Figure 5 Quantitative analyses of OsUBC13 expression. a Expression of OsUBC13 (LOC_Os01g48280) during rice development. Samples were taken from different developmental stages as indicated and relative transcript levels of the entire transcriptome were determined by microarray analysis. The data is retrieved from Genevestigator (www.genevestigator.com). b Relative OsUbc13 protein level in rice seedlings after treatment with various stresses, two weeks after germination rice seedlings were treated with the given biotic or abiotic agent for the predetermined time as indicated on top of each panel and total proteins were extracted from the whole plants. The cellular OsUbc13 was detected by western bolt analysis using the anti-hUbc13 mAb 4E11 (Andersen et al. 2005). Each lane contains $20 \mu \mathrm{g}$ total protein and the most abundant protein band as visualized by Coomassie brilliant blue staining serves as a loading control (the bottom image of each panel). CK, untreated seedlings; Ct, cisplatin.

densitometry (data not shown), no obvious alteration was observed by any given treatments under our experimental conditions.

\section{Discussion}

While sunlight is absolutely necessary for photosynthesis by land plants, the threats of solar UV radiation as well as other genotoxic chemicals are also critical to plants survival due to their sessile living style. As the major food resources for human beings, crops may severely lose yield and quality due to the above stresses, which compromise global food security. In this study, we isolated and characterized the rice $U B C 13$ gene, which is believed to be the first reported crop gene involved in the DNA damage-tolerance pathway. Our in vitro studies confirmed that the highly conserved OsUbc13 protein could physically interact with the cognate E2 variants from yeast and human. Functional examination revealed that OsUBC13 could restore cellular activities of the yeast $u b c 13$ null mutant to limit spontaneous mutagenesis and provide resistance to DNA-damaging agents. Since yeast $U B C 13$ plays an essential role in mediating error-free DDT (Broomfield et al. 1998), these results suggest that OsUBC13 is involved in the error-free DDT pathway in rice as well, although definitive evidence has to wait for the characterization of $U B C 13$ knockdown or knockout rice plants. It is noticed that rice may be a more suitable experimental plant model than other model plants including Arabidopsis, since it contains only one predicted $U B C 13$ gene, while Arabidopsis contains two highly conserved and most likely redundant $U B C 13$ genes (Wen et al. 2006). Furthermore, since the DDT pathway appears to be conserved from yeast to humans (Andersen et al. 2005), this study may serve as a gateway to understanding DDT mechanisms in rice in an attempt to enhance its resistance to this major environment stress.

Ubc13 is the only known E2 that catalyzes K63-linked polyubiquitination. This catalytic activity is dependent on its interaction with an E2 variant (Uev) and Ubc13 associated with different Uevs is thought to mediate different cellular processes (Andersen et al. 2005). In S. cerevisiae, Ubc13 has only one Uev partner, Mms2, and the Ubc13Mms2 heterodimer is required for the K63 polyubiquitination of PCNA (Hoege et al. 2002). In human cells, two Ubc13-associated Uevs are found (Xiao et al. 1998), and they appear to play different roles (Andersen et al. 2005): the Ubc13-hMms2 complex is required for DNAdamage response, while the Ubc13-Uev1A complex is required for the NF- $\mathrm{kB}$ signaling pathway (Deng et al. 2000) through activation of NEMO (Zhou et al. 2004) 
and/or TAK1 (Wang et al. 2001). In this study, we found that OsUbc13 forms stable complexes with both hMms2 and Uev1A, and could catalyze K63-linked poly-Ub chains in vitro with either Uev1A or hMms2. Interestingly, Arabidopsis contains four UEV1 genes; at least one of them $(U E V 1 D)$ is involved in DNA-damage response, while at least one (UEV1A) is not required for this response (Wen et al. 2008). Since the rice genome also contains multiple UEV1 homologs (Y.Z. and W.X., data not shown), it is conceivable that rice Ubc13 associates with different Uev1s to catalyze K63-linked poly-Ub chains. These different heterodimers may interact with different E3s, target proteins and hence are involved in various stress responses including, but not limited to, DNA-damage tolerance. Indeed, Ubc13-mediated ubiquitination has been reported to be involved in cellular processes such as neurodegeneration (Doss-Pepe et al. 2005; Lim et al. 2005; Muralidhar and Thomas 1993; Oh et al. 1994), homologous recombination (Doil et al. 2009; Huen et al. 2007; Mailand et al. 2007; Stewart et al. 2009), innate immunity (Deng et al. 2000; Zhou et al. 2004), cell cycle checkpoint (Bothos et al. 2003; Laine et al. 2006; Wen et al. 2012), as well as development (Yin et al. 2007) and iron metabolism ( $\mathrm{Li}$ and Schmidt 2010) in Arabidopsis. Some of these processes may not necessarily require a Uev (Huen et al. 2008) or involve K63linked polyubiquitination.

K63-linked polyubiquitination process is primarily involved in stress responses, within which the E2 complex plays a critical role. It is reasonable to assume that the Ubc13-Uev activity is tightly regulated in response to stress. In S. cerevisiae, UBC13 is transcriptionally regulated in response to DNA damage. In higher eukaryotes, because Ubc13 is involved in multiple cellular processes, and whose specificity is largely determined by the cognate Uev, regulation of Uev activity would be evolutionarily preferred. Indeed, there is little experimental evidence on altered Ubc13 activity in mammalian cells, while cellular Uev levels appear to fluctuate in different tissues and in response to environmental stresses (Fritsche et al. 1997; Ma et al. 1998; Sancho et al. 1998). Of great interest is the observation that transcript levels of $U B C 13$ genes in Arabidopsis and rice are relatively stable regardless of plant development, tissue distribution and response to stresses, indicating that $U B C 13$ is a housekeeping gene in plants. In sharp contrast, the expression of plant UEV1 genes fluctuate under the same experimental conditions, suggesting that plants achieve the control of K63 polyubiquitination through differential regulation of $U E V 1$ genes, or that Uevs serve as a regulatory subunit for K63 ubiquitination. This molecular evolution further testifies that in higher eukaryotes, K63 ubiquitination is involved in multiple cellular processes.
Since transcriptional regulation is only one of several means of controlling target gene activity, in this study we attempted to assess the cellular Ubc13 protein level under different stress conditions. Western blot analysis by using an anti-hUbc13 monoclonal antibody further confirms that $U B C 13$ is a candidate housekeeping gene. Hence, we recommend Ubc13 to be a suitable candidate to serve as an internal reference for rice-related research because it fulfills the following criteria: (a) it is a highly expressed gene; (b) its expression level is remarkably constant under various growth stages, tissues and experimental conditions; (c) there is only one copy of the homologous gene; and (d) the monoclonal antibody 4E11 used in this study is commercially available.

\section{Conclusions}

The present study describes the isolation and functional characterization of the rice $U B C 13$ (OsUBC13) gene and its product. Our observations collectively suggest that Ubc13-mediated K63 polyubiquitination is conserved in rice, and that OsUBC13 may be involved in DNA damage tolerance as well as other cellular stress responses. Furthermore, bioinformatic analysis and our experimental data suggest that OsUBC13 is a housekeeping gene, and that its expression can be utilized as an internal control for other rice-related investigations.

\section{Methods}

\section{Plant materials and yeast cell culture}

Rice (Oryza sativa L. cv. Japonaca) seeds were surface sterilized with $2 \% \mathrm{NaClO}$ for $30 \mathrm{~min}$ after pre-wash by sterilized distilled water, followed by washing seven times in sterilized water. The sterilized rice seeds were plated in $1 / 2$ Murashige and Skoog (MS) plates containing MS with $2.2 \mathrm{~g} / \mathrm{l}$ minimal organics (Sigma M6899), $10 \mathrm{~g} / \mathrm{l}$ sucrose and 1\% agar (BD 214010), and vertically cultured in a growth chamber $\left(16 \mathrm{~h}\right.$ light $/ 8 \mathrm{~h}$ dark and $30^{\circ} \mathrm{C}$ constant). Two weeks after seed germination, rice seedlings were transferred to $1 / 2 \mathrm{MS}$ solution for an additional 1day incubation. For ABA (final concentration $50 \mu \mathrm{M}$ ), MMS (final concentration of $0.175 \%$ ) and cisplatin (final concentration $16 \mu \mathrm{M}$ ) treatments, stock solutions were added to the nutrient solution to the predetermined final concentration. For SA and meJA treatments, SA (5 mM in water) and meJA (0.1 $\mathrm{mM}$ in $0.1 \%$ ethanol) were sprayed onto the rice shoot. For ABA, SA, meJA and $\mathrm{H}_{2} \mathrm{O}_{2}$ treatments, the seedlings were harvested after 8 hours. For MMS and cisplatin treatments, seedlings were harvested after 12 hours.

The haploid yeast strains used in this study are listed in Additional file 4: Table S1. Yeast cells were grown at $30^{\circ} \mathrm{C}$ in either rich YPD or in a synthetic dextrose (SD) medium $(0.67 \%$ Bacto-yeast nitrogen base without amino acids, $2 \%$ glucose) supplemented with necessary 
nutrients as recommended (Sherman et al. 1983). For solid plates, $2 \%$ agar was added to either YPD or SD medium prior to autoclaving. Yeast cells were transformed using a LiAc method as described (Ito et al. 1983). The source and preparation of $u b c 13 \Delta:: H I S 3$ cassettes was as previously described (Xiao et al. 2000).

\section{Cloning rice UBC13 CDNA and plasmid construction}

To clone the full-length open reading frame (ORF) of OsUBC13, total RNA was isolated from rice seedlings by using TRIzol reagents (Invitrogen, Carlsbad, CA, USA) for RT-PCR with the RevertAid First Strand cDNA Synthesis Kit (Fermentas) following the manufacturer's protocols. OsUbc13 specific primers are as follow: 5 '-aatccggaattc ATGGCCAACAGCAACCTC-3' and 5'-atacgcgtcgacT TATGCACCGCTGGC-3'. The forward primer contains an EcoRI restriction site and the reverse primer contains a SalI site, as underlined. The PCR product of OsUBC13 ORF was cloned into yeast two-hybrid vectors pGBT9Bg and pGAD424Bg, which were derived from pGBT9 and pGAD424 (Bartel and Fields 1995), respectively.

\section{Yeast two-hybrid analysis}

The yeast two-hybrid strain PJ69-4A (James et al. 1996) was used for this assay. The co-transformation, selection and two-hybrid detection steps were as previously described (Wen et al. 2006).

\section{Recombinant protein purification and ubiquitination assay}

The OsUbc13 ORF was isolated from pGBT-OsUbc13 and cloned into pET-30a. The resulting pET-OsUbc13 was transformed into BL21 CodonPlus (DE3)-RIL cells. The $\mathrm{His}_{6}$-OsUbc13 fusion protein was purified following a previously published protocol (Anderson et al. 2008). Meanwhile, GST, GST-hUev1A and GST-hMms2 were produced and purified as previously described (Andersen et al. 2005). For an in vitro ubiquitination assay, the 20$\mu \mathrm{l}$ reaction mixture contained $225 \mathrm{nM}$ E1 enzyme, $450 \mu \mathrm{M}$ Ub, $1 \mathrm{mM}$ Mg-ATP, $1 \mu \mathrm{M}$ Ubc13, and $1 \mu \mathrm{M}$ Uev1A in the supplied reaction buffer (Boston Biochem). The K63R and K48R mutant Ub proteins were also purchased from Boston Biochem. The conjugation reactions were performed at $37^{\circ} \mathrm{C}$ for $2 \mathrm{hr}$. Samples were subjected to SDS-PAGE (12\%), and Ub and poly-Ub were detected through protein gel blots using polyclonal rabbit anti-Ub antibodies (Sigma-Aldrich).

\section{GST pull-down assay}

The BL21 competent cells were transformed with either pGEX6p-1, pGEX-hMms2 or pGEX-hUev1A alone, or co-transformed with pET-OsUbc13. The whole cell extracts were incubated with Glutathione Sepharose 4B Microspin ${ }^{\mathrm{TM}}$ beads in $4^{\circ} \mathrm{C}$ for $2 \mathrm{hr}$. The beads were collected by centrifugation and washed with a lysis buffer 5 times. Finally, beads were boiled with loading buffer and analyzed on a $12 \%$ SDS-PAGE gel.

\section{Rice protein extraction and western blot analysis}

Two-week-old rice seedlings were homogenized in liquid nitrogen. Total protein was extracted using a buffer containing $50 \mathrm{mM}$ Tris- $\mathrm{HCl}$ pH8.0, $0.3 \mathrm{M} \mathrm{NaCl}, 2 \mathrm{mM}$ EDTA, 10\% Glycerol, 0.1\% TritonX-100, 10 mM PMSF, $3 \mathrm{mM}$ DDT and $1 / 5$ tablet per $10 \mathrm{ml}$ protease inhibitor (Roche). The extract was centrifuged at $16,000 \mathrm{~g}$ for $10 \mathrm{~min}$ at $4^{\circ} \mathrm{C}$. The supernatant was then transferred to a new tube and centrifuged twice prior to SDS/PAGE analysis. The OsUbc13 protein in each sample was detected by western bolt using the anti-hUbc13 monoclonal antibody 4E11 (Andersen et al. 2005).

\section{Yeast cell survival assays}

Yeast strain HK578-10D and its isogenic $u b c 13 \Delta$ single mutant transformed with pGAD424 or pGAD-OsUbc13, were used in this assay. The yeast liquid killing and serial dilution assays were performed as previously described (Barbour et al. 2006; Wang et al. 2011). For the serial dilution assay, several doses for each DNA-damaging agent were employed and only one representative dose is presented.

\section{Spontaneous mutagenesis assay}

Yeast strain DBY747 and its isogenic $u b c 13 \Delta$ derivative WXY849 bear a trp1-289 amber mutation that can be reverted to $\operatorname{Trp}^{+}$by several different mutation events (Xiao and Samson 1993). WXY849 was transformed with pGAD-OsUbc13 and the spontaneous mutagenesis assay was performed as described previously (Anderson et al. 2008).

\section{Additional files}

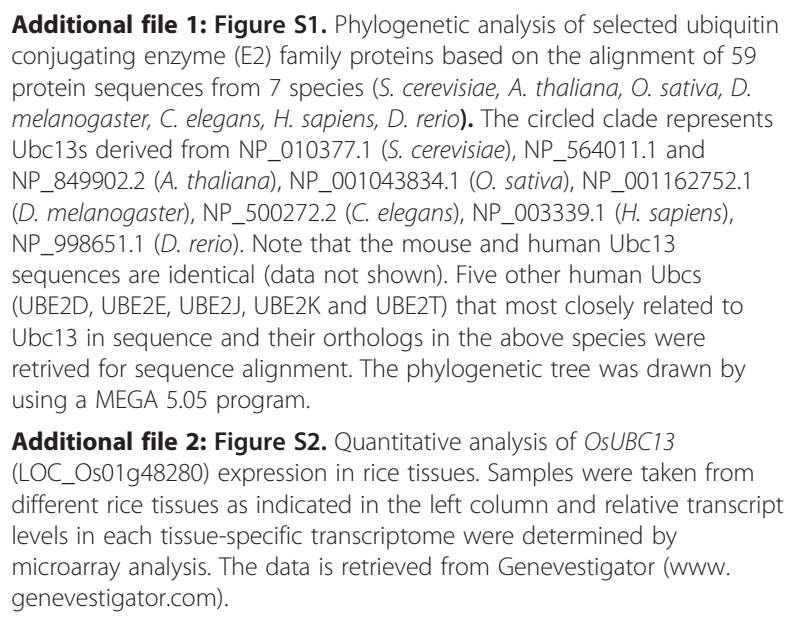

Additional file 1: Figure S1. Phylogenetic analysis of selected ubiquitin conjugating enzyme (E2) family proteins based on the alignment of 59 protein sequences from 7 species (S. cerevisiae, A. thaliana, O. sativa, D. melanogaster, C. elegans, H. sapiens, D. rerio). The circled clade represents Ubc13s derived from NP_010377.1 (S. cerevisiae), NP_564011.1 and NP_849902.2 (A. thaliana), NP_001043834.1 (O. sativa), NP_001162752.1 (D. melanogaster), NP_500272.2 (C. elegans), NP_003339.1 (H. sapiens), NP_998651.1 (D. rerio). Note that the mouse and human Ubc13 sequences are identical (data not shown). Five other human Ubcs (UBE2D, UBE2E, UBE2J, UBE2K and UBE2T) that most closely related to Ubc13 in sequence and their orthologs in the above species were retrived for sequence alignment. The phylogenetic tree was drawn by using a MEGA 5.05 program.

Additional file 2: Figure S2. Quantitative analysis of OsUBC13 (LOC_Os01g48280) expression in rice tissues. Samples were taken from different rice tissues as indicated in the left column and relative transcript levels in each tissue-specific transcriptome were determined by microarray analysis. The data is retrieved from Genevestigator (www. genevestigator.com).

Additional file 3: Figure S3. Quantitative analysis of OsUBC13 (LOC_Os01g48280) expression in rice under different stress conditions. 
Samples were taken from rice tissues after the plants were treated as indicated in the left column. Relative expression levels were determined by microarray analysis. The resulting data were compiled and retrieved from Genevestigator (www.genevestigator.com), and displayed in log2 scale in the middle column. The actual fold change values upon stress treatment are given in the right column.

Additional file 4: Table S1. Saccharomyces cerevisiae strains.

\section{Competing interests}

The authors declare that they have no competing interests.

\section{Authors' contributions}

$Y Z$ cloned the OsUBC13 gene, performed bioinformatics analyses and drafted the manuscript. YZ, QW, CX and ML conducted functional characterization. YZ and RW performed the ubiquitination assay. WX conceived the proposal, generated financial support for the study and corrected the final manuscript. All authors read and approved the final manuscript.

\section{Acknowledgments}

The authors wish to thank Guang Yang, Xin Xu and Amanda Lambrecht for technical assistance and other Xiao laboratory members for helpful discussion. This work was supported by an operating grant (No. KZ201110028037) from Beijing Education Committee.

Received: 13 March 2012 Accepted: 26 July 2012

Published: 8 September 2012

\section{References}

Andersen PL, Zhou H, Pastushok L, Moraes T, McKenna S, Ziola B, Ellison MJ, Dixit VM, Xiao W (2005) Distinct regulation of Ubc13 functions by the two ubiquitinconjugating enzyme variants Mms2 and Uev1 A. J Cell Biol 170:745-755

Anderson HJ, Vonarx EJ, Pastushok L, Nakagawa M, Katafuchi A, Gruz P, Di Rubbo A, Grice DM, Osmond MJ, Sakamoto AN, Nohmi T, Xiao W, Kunz BA (2008) Arabidopsis thaliana $Y$-family DNA polymerase $\eta$ catalyses translesion synthesis and interacts functionally with PCNA2. Plant J 55:895-908

Ardley HC, Robinson PA (2005) E3 ubiquitin ligases. Essays Biochem 41:15-30

Barbour L, Ball LG, Zhang K, Xiao W (2006) DNA damage checkpoints are involved in postreplication repair. Genetics 174:1789-1800

Bartel PL, Fields S (1995) Analyzing protein-protein interactions using two-hybrid system. Methods Enzymol 254:241-263

Bothos J, Summers MK, Venere M, Scolnick DM, Halazonetis TD (2003) The Chfr mitotic checkpoint protein functions with Ubc13-Mms2 to form Lys63-linked polyubiquitin chains. Oncogene 22:7101-7107

Broomfield S, Chow BL, Xiao W (1998) MMS2, encoding a ubiquitin-conjugatingenzyme-like protein, is a member of the yeast error-free postreplication repair pathway. Proc Natl Acad Sci USA 95:5678-5683

Broomfield S, Hryciw T, Xiao W (2001) DNA postreplication repair and mutagenesis in Saccharomyces cerevisiae. Mutat Res 486:167-184

Brusky J, Zhu Y, Xiao W (2000) UBC13, a DNA-damage-inducible gene, is a member of the error-free postreplication repair pathway in Saccharomyces cerevisiae. Curr Genet 37:168-174

Burroughs AM, Jaffee M, lyer LM, Aravind L (2008) Anatomy of the E2 ligase fold: implications for enzymology and evolution of ubiquitin/Ub-like protein conjugation. J Struct Biol 162:205-218

Chen IP, Mannuss A, Orel N, Heitzeberg F, Puchta H (2008) A homolog of SCRAD5 is involved in DNA repair and homologous recombination in Arabidopsis. Plant Physiol 146:1786-1796

Curtis MJ, Hays JB (2007) Tolerance of dividing cells to replication stress in UVBirradiated Arabidopsis roots: requirements for DNA translesion polymerases $\eta$ and $\zeta$. DNA Repair 6:1341-1358

Deng L, Wang C, Spencer E, Yang L, Braun A, You J, Slaughter C, Pickart C, Chen ZJ (2000) Activation of the IkB kinase complex by TRAF6 requires a dimeric ubiquitin-conjugating enzyme complex and a unique polyubiquitin chain. Cell 103:351-361

Deshaies RJ, Joazeiro CA (2009) RING domain E3 ubiquitin ligases. Annu Rev Biochem 78:399-434

Doil C, Mailand N, Bekker-Jensen S, Menard P, Larsen DH, Pepperkok R, Ellenberg J, Panier S, Durocher D, Bartek J, Lukas J, Lukas C (2009) RNF168 binds and amplifies ubiquitin conjugates on damaged chromosomes to allow accumulation of repair proteins. Cell 136:435-446

Doss-Pepe EW, Chen L, Madura K (2005) a-Synuclein and Parkin contribute to the assembly of ubiquitin lysine 63-linked multiubiquitin chains. J Biol Chem 280:16619-16624

Fields S, Song O (1989) A novel genetic system to detect protein-protein interactions. Nature 340:245-246

Fritsche J, Rehli M, Krause SW, Andreesen R, Kreutz M (1997) Molecular cloning of a 1a,25-dihydroxyvitamin D3-inducible transcript (DDVit 1) in human blood monocytes. Biochem Biophys Res Commun 235:407-412

Garcia-Ortiz MV, Ariza RR, Hoffman PD, Hays JB, Roldan-Arjona T (2004) Arabidopsis thaliana AtPOLK encodes a DinB-like DNA polymerase that extends mispaired primer termini and is highly expressed in a variety of tissues. Plant J 39:84-97

Hershko A, Ciechanover A (1998) The ubiquitin system. Annu Rev Biochem 67:425-479

Hochstrasser M (1996) Ubiquitin-dependent protein degradation Annu Rev Genet 30:405-439

Hoege C, Pfander B, Moldovan GL, Pyrowolakis G, Jentsch S (2002) RAD6dependent DNA repair is linked to modification of PCNA by ubiquitin and SUMO. Nature 419:135-141

Hofmann RM, Pickart CM (1999) Noncanonical MMS2-encoded ubiquitinconjugating enzyme functions in assembly of novel polyubiquitin chains for DNA repair. Cell 96:645-653

Hofmann RM, Pickart CM (2001) In vitro assembly and recognition of Lys-63 polyubiquitin chains. J Biol Chem 276:27936-27943

Hruz T, Laule O, Szabo G, Wessendorp F, Bleuler S, Oertle L, Widmayer P. Gruissem W, Zimmermann P (2008) Genevestigator v3: a reference expression database for the meta-analysis of transcriptomes. Advances in bioinformatics 2008:420747

Huen MS, Grant R, Manke I, Minn K, Yu X, Yaffe MB, Chen J (2007) RNF8 transduces the DNA-damage signal via histone ubiquitylation and checkpoint protein assembly. Cell 131:901-914

Huen MS, Huang J, Yuan J, Yamamoto M, Akira S, Ashley C, Xiao W, Chen J (2008) Noncanonical E2 variant-independent function of UBC13 in promoting checkpoint protein assembly. Mol Cell Biol 28:6104-6112

Ito H, Fukuda Y, Murata K, Kimura A (1983) Transformation of intact yeast cells treated with alkali cations. J Bacteriol 153:163-168

James P, Halladay J, Craig EA (1996) Genomic libraries and a host strain designed for highly efficient two-hybrid selection in yeast. Genetics 144:1425-1436

Kraft E, Stone SL, Ma L, Su N, Gao Y, Lau OS, Deng XW, Callis J (2005) Genome analysis and functional characterization of the E2 and RING-type E3 ligase ubiquitination enzymes of Arabidopsis. Plant Physiol 139:1597-1611

Laine A, Topisirovic I, Zhai D, Reed JC, Borden KL, Ronai Z (2006) Regulation of p53 localization and activity by Ubc13. Mol Cell Biol 26:8901-8913

Li W, Schmidt W (2010) A lysine-63-linked ubiquitin chain-forming conjugase, UBC13, promotes the developmental responses to iron deficiency in Arabidopsis roots. Plant J 62:330-343

Lim KL, Chew KC, Tan JM, Wang C, Chung KK, Zhang Y, Tanaka Y, Smith W, Engelender S, Ross CA, Dawson VL, Dawson TM (2005) Parkin mediates nonclassical, proteasomal-independent ubiquitination of synphilin-1: implications for Lewy body formation. J Neurosci 25:2002-2009

Ma L, Broomfield S, Lavery C, Lin SL, Xiao W, Bacchetti S (1998) Up-regulation of CIR1/CROC1 expression upon cell immortalization and in tumor-derived human cell lines. Oncogene 17:1321-1326

Mailand N, Bekker-Jensen S, Faustrup H, Melander F, Bartek J, Lukas C, Lukas J (2007) RNF8 ubiquitylates histones at DNA double-strand breaks and promotes assembly of repair proteins. Cell 131:887-900

McKenna S, Spyracopoulos L, Moraes T, Pastushok L, Ptak C, Xiao W, Ellison M. (2001) Noncovalent interaction between ubiquitin and the human DNA repair protein Mms2 is required for Ubc13-mediated polyubiquitination. J Biol Chem 276:40120-40126

Muralidhar MG, Thomas JB (1993) The Drosophila bendless gene encodes a neural protein related to ubiquitin-conjugating enzymes. Neuron 11:253-266

Oh CE, McMahon R, Benzer S, Tanouye MA (1994) bendless, a Drosophila gene affecting neuronal connectivity, encodes a ubiquitin-conjugating enzyme homolog. J Neurosci 14:3166-3179 
Papouli E, Chen S, Davies AA, Huttner D, Krejci L, Sung P, Ulrich HD (2005) Crosstalk between SUMO and ubiquitin on PCNA is mediated by recruitment of the helicase Srs2p. Mol Cell 19:123-133

Pastushok L, Moraes TF, Ellison MJ, Xiao W (2005) A single Mms2 "key" residue insertion into a Ubc13 pocket determines the interface specificity of a human Lys63 ubiquitin conjugation complex. J Biol Chem 280:17891-17900

Pastushok L, Xiao W (2004) DNA postreplication repair modulated by ubiquitination and sumoylation. Adv Protein Chem 69:279-306

Pfander B, Moldovan GL, Sacher M, Hoege C, Jentsch S (2005) SUMO-modified PCNA recruits Srs 2 to prevent recombination during $S$ phase. Nature 436:428-433

Pickart CM (2000) Ubiquitin in chains. Trends Biochem Sci 25:544-548

Pickart CM, Fushman D (2004) Polyubiquitin chains: polymeric protein signals. Curr Opin Chem Biol 8:610-616

Rotin D, Kumar S (2009) Physiological functions of the HECT family of ubiquitin ligases. Nat Rev Mol Cell Biol 10:398-409

Sakamoto A, Lan VT, Hase Y, Shikazono N, Matsunaga T, Tanaka A (2003) Disruption of the AtREV3 gene causes hypersensitivity to ultraviolet B light and gamma-rays in Arabidopsis: implication of the presence of a translesion synthesis mechanism in plants. Plant Cell 15:2042-2057

Sanada T, Kim M, Mimuro H, Suzuki M, Ogawa M, Oyama A, Ashida H, Kobayashi T, Koyama T, Nagai S, Shibata Y, Gohda J, Inoue J, Mizushima T, Sasakawa C (2012) The Shigella flexneri effector Ospl deamidates UBC13 to dampen the inflammatory response. Nature 483:623-626

Sancho E, Vila MR, Sanchez-Pulido L, Lozano JJ, Paciucci R, Nadal M, Fox M, Harvey C, Bercovich B, Loukili N, Ciechanover A, Lin SL, Sanz F, Estivill X, Valencia A, Thomson TM (1998) Role of UEV-1, an inactive variant of the E2 ubiquitin-conjugating enzymes, in in vitro differentiation and cell cycle behavior of HT-29-M6 intestinal mucosecretory cells. Mol Cell Biol 18:576-589

Sherman F, Fink GR, Hicks J (1983) Methods in Yeast Genetics. Cold Spring Harbor Laboratory Press, Cold Spring Harbor, NY

Stelter P, Ulrich HD (2003) Control of spontaneous and damage-induced mutagenesis by SUMO and ubiquitin conjugation. Nature 425:188-191

Stewart GS, Panier S, Townsend K, Al-Hakim AK, Kolas NK, Miller ES, Nakada S, Ylanko J, Olivarius S, Mendez M, Oldreive C, Wildenhain J, Tagliaferro A, Pelletier L, Taubenheim N, Durandy A, Byrd PJ, Stankovic T, Taylor AM, Durocher D (2009) The RIDDLE syndrome protein mediates a ubiquitindependent signaling cascade at sites of DNA damage. Cell 136:420-434

Takahashi S, Sakamoto A, Sato S, Kato T, Tabata S, Tanaka A (2005) Roles of Arabidopsis AtREV1 and AtREV7 in translesion synthesis. Plant Physiol 138:870-881

Ulrich HD, Jentsch S (2000) Two RING finger proteins mediate cooperation between ubiquitin-conjugating enzymes in DNA repair. EMBO J 19:3388-3397

van Wijk SJL, Timmers HTM (2009) The family of ubiquitin-conjugating enzymes (E2s): deciding between life and death of proteins. FASEB J 24:981-993

VanDemark AP, Hofmann RM, Tsui C, Pickart CM, Wolberger C (2001) Molecular insights into polyubiquitin chain assembly: crystal structure of the Mms2/Ubc13 heterodimer. Cell 105:711-720

Vierstra RD (2009) The ubiquitin-26 S proteasome system at the nexus of plant biology. Nat Rev Mol Cell Biol 10:385-397

Wang C, Deng L, Hong M, Akkaraju GR, Inoue J, Chen ZJ (2001) TAK1 is a ubiquitin-dependent kinase of MKK and IKK. Nature 412:346-351

Wang S, Wen R, Shi X, Lambrecht A, Wang H, Xiao W (2011) RAD5a and REV3 function in two alternative pathways of DNA-damage tolerance in Arabidopsis. DNA Repair 10:620-628

Wen R, Li J, Xu X, Cui Z, Xiao W (2012) Zebrafish Mms2 promotes K63-linked polyubiquitination and is involved in p53-mediated DNA-damage response. DNA repair 11:157-166

Wen R, Newton L, Li G, Wang H, Xiao W (2006) Arabidopsis thaliana UBC13: implication of error-free DNA damage tolerance and Lys63-linked polyubiquitylation in plants. Plant Mol Biol 61:241-253

Wen R, Torres-Acosta JA, Pastushok L, Lai XQ, Pelzer L, Wang H, Xiao W (2008) Arabidopsis UEV1D promotes lysine-63-linked polyubiquitination and is involved in DNA damage response. Plant Cell 20:213-227

Wooff J, Pastushok L, Hanna M, Fu Y, Xiao W (2004) The TRAF6 RING finger domain mediates physical interaction with Ubc13. FEBS Lett 566:229-233

Xiao W, Chow BL, Broomfield S, Hanna M (2000) The Saccharomyces cerevisiae RAD6 group is composed of an error-prone and two error-free postreplication repair pathways. Genetics 155:1633-1641
Xiao W, Lin SL, Broomfield S, Chow BL, Wei YF (1998) The products of the yeast MMS2 and two human homologs ( $h M M S 2$ and CROC-1) define a structurally and functionally conserved Ubc-like protein family. Nucleic Acids Res 26:3908-3914

Xiao W, Samson L (1993) In vivo evidence for endogenous DNA alkylation damage as a source of spontaneous mutation in eukaryotic cells. Proc Natl Acad Sci USA 90:2117-2121

Yin XJ, Volk S, Ljung K, Mehlmer N, Dolezal K, Ditengou F, Hanano S, Davis SJ, Schmelzer E, Sandberg G, Teige M, Palme K, Pickart C, Bachmair A (2007) Ubiquitin lysine 63 chain forming ligases regulate apical dominance in Arabidopsis. Plant Cell 19:1898-1911

Zhou H, Wertz I, O'Rourke K, Ultsch M, Seshagiri S, Eby M, Xiao W, Dixit VM (2004) BCl10 activates the NF-KB pathway through ubiquitination of NEMO. Nature 427:167-171

doi:10.1186/1939-8433-5-24

Cite this article as: Zang et al:: Rice UBC13, a candidate housekeeping gene, is required for K63-linked polyubiquitination and tolerance to DNA damage. Rice 2012 5:24.

\section{Submit your manuscript to a SpringerOpen ${ }^{\circ}$ journal and benefit from:}

- Convenient online submission

- Rigorous peer review

- Immediate publication on acceptance

- Open access: articles freely available online

- High visibility within the field

- Retaining the copyright to your article

Submit your next manuscript at $>$ springeropen.com 